\title{
RAP: A Novel Approach to the Rapid and Highly Sensitive Detection of Respiratory Viruses
}

\author{
Guohao Fan ${ }^{1+}$, Ruiqing Zhang ${ }^{1 \dagger}$, Xiaozhou He ${ }^{1}$, Fengyu Tian ${ }^{1}$, Mingzhu Nie ${ }^{1}$, Xinxin Shen ${ }^{1 *}$ \\ and Xuejun $\mathrm{Ma}^{1,2 *}$ \\ ${ }^{1}$ National Institute for Viral Disease Control and Prevention, Chinese Center for Disease Control and Prevention, Beijing, China, \\ ${ }^{2}$ Center for Biosafety Mega-Science, Chinese Academy of Sciences, Wuhan, China
}

OPEN ACCESS

Edited by: Can Dincer

University of Freiburg, Germany

Reviewed by: Kang Mao, Institute of Geochemistry (CAS), China Bicheng Zhu,

The University of Auckland, New Zealand

${ }^{*}$ Correspondence:

Xinxin Shen

x616815@126.com

Xuejun Ma

maxj@ivdc.chinacdc.cn

${ }^{\dagger}$ These authors have contributed equally to this work

Specialty section: This article was submitted to Biosensors and Biomolecular

Electronics,

a section of the journal Frontiers in Bioengineering and

Biotechnology

Received: 29 August 2021 Accepted: 13 October 2021 Published: 05 November 2021

Citation:

Fan G, Zhang R, He X, Tian F, Nie M, Shen $X$ and Ma X (2021) RAP: A Novel Approach to the Rapid and Highly Sensitive Detection of Respiratory Viruses.

Front. Bioeng. Biotechnol. 9:766411. doi: 10.3389/fbioe.2021.766411
Recombinase aided amplification (RAA) is an emerging isothermal amplification method used for detecting various pathogens. However, RAA requires a complex and long probe to ensure high sensitivity during fluorescence assay. TaqMan probe used for quantitative PCR (qPCR) is simple and universal. Herein, we developed a new approach for detecting nucleic acids of pathogens, known as RAP (Recombinase aided PCR). The method combines RAA and qPCR to ensure a rapid and highly sensitive detection using a conventional qPCR device. RAP is a two-stage amplification process performed in a single tube within 1 hour. The method involves an RAA reaction for 10 min at $39^{\circ} \mathrm{C}$ (first stage) followed by 15 cycles of qPCR (second stage). Using human adenovirus 3 (HADV3) and human adenovirus 7 (HADV7) plasmids, the sensitivities of RAP assays for detecting HADV3 and HADV7 were 6 and 17 copies per reaction, respectively. The limit of RAP detection was at least 16 -fold lower than the corresponding $\mathrm{qPCR}$, and no-cross reaction with other respiratory viruses was observed. The results of RAP analysis revealed $100 \%$ consistency with qPCR assay. This study shows that RAP assay is a rapid, specific, and highly sensitive detection method with a potential for clinical and laboratory application.

Keywords: respiratory viruses, qPCR, RAA, highly sensitive, clinical detection

\section{INTRODUCTION}

Techniques for analyzing nucleic acids in samples are widely used for quantitative and qualitative detection of pathogens in clinical laboratories (Riley, 2018; Kellner et al., 2019; Pumford et al., 2020; Corman et al., 2020; Paul et al., 2020). Nucleic acid amplification is typically carried out with polymerase chain reaction (PCR) and various isothermal amplification methods (Waggoner et al., 2016), such as recombinase polymerase amplification (RPA) (Lobato and O'Sullivan, 2018), recombinase aided amplification (RAA) (Qi et al., 2019), rolling-circle amplification (RCA) (Marano et al., 2020), loop-mediated isothermal amplification (LAMP) (Zhu et al., 2020) and helicase dependent amplification (HDA) (Liu et al., 2020). PCR has the advantages of simple primers and probe design and real time fluorescence quantitative detection, however it is time-consuming and needs to be improved in the sensitivity. The isothermal amplification methods have the advantages of high sensitivity and rapidity, but the designs of multiple primers (LAMP) or long probes (RAA/RPA) are difficult (Lobato and O'Sullivan, 2018; Qi et al., 2019; Zhu et al., 2020). RAA is an emerging isothermal amplification assay used to detect pathogens at $37-42^{\circ} \mathrm{C}$. RAA is highly efficient and can amplify tiny amounts of DNA fragments in 5-15 min. It uses a fluorescence probe for real-time qualitative detection. Fluorescence RAA has been successfully applied for rapid 
detection of various bacterial and viral pathogens and single nucleotide polymorphism (SNP) (Duan et al., 2018; Fan et al., 2019; Qi et al., 2019; Zhang et al., 2019; Chen et al., 2020; Tu et al., 2020; Wang et al., 2020). However, the length requirement of the RAA probe is $46-52 \mathrm{bp}$, which is significantly longer than the TaqMan probe. Notably, long RAA probes are difficult to design because of sequence specificity requirement. Moreover, the introduction of degenerated bases in the probe can reduce the RAA sensitivity and result in non-specific amplification. Quantitative PCR (qPCR) is the "gold standard" of nucleic acid test for many pathogens (Bonroy et al., 2007; Mahony et al., 2011; Qiu et al., 2018). The sensitivity of qPCR can be improved by nest-PCR (N-PCR). Traditional two-step N-PCR exhibit higher sensitivity but the test samples are prone to contamination. The one-tube N-PCR developed in our laboratory significantly reduced the risk of contamination but was consumed more time than qPCR (Feng et al., 2018; Zhang et al., 2020; Zhao et al., 2020). Cognizant of the strengths and weaknesses of the methods mentioned above, there is a need to integrate the strengths of the various techniques and develop next-generation technology for rapid and highly sensitive detection.

Previous research reported a dubbed Penn-RAMP, a combination of RPA and LAMP, to improve the sensitivity of RPA and LAMP alone (Song et al., 2017). In this study, we integrated the distinctive features of RAA rapidity and the probe universality of qPCR and developed a novel approach for detecting pathogens, known as RAP (Recombinase aided PCR). The principle of RAP methods involves enriching tiny amounts of target DNA fragments using RAA for 10 min then amplifying the enriched templates using GPCR with 15 thermal cycles. Furthermore, we explored the physical isolation strategy using respiratory viruses (ADV3 and ADV7) as test samples and managed to complete consecutive nucleic acid amplification and detection in a single tube within 1 hour.

\section{METHODS}

\section{Samples Collection}

In total, 200 stock clinical samples (150 nasopharyngeal aspirate and 50 throat swabs) were collected from Hunan Provincial Center for Disease Control and Prevention and the Children Hospital of Hebei Province, China. These samples were stored at $-80^{\circ} \mathrm{C}$. Of note, these samples were previously tested for the presence of respiratory viruses by GeXP-based multiplex PCR/ RT-PCR assay (Wang et al., 2019). The clinical samples comprised 30 human adenovirus 3 (HADV3) and 30 human adenovirus 7 (HADV7) positive samples. The rest of the samples were either positive or negative for other respiratory pathogens.

\section{Nucleic Acid Extraction}

Total DNA and RNA were extracted from $200 \mu \mathrm{L}$ of each clinical sample using Viral RNA/DNA Isolation Kit (Tianlong, Suzhou, China) according to the manufacturer's instructions. The extracted nucleic acids were eluted in $50 \mu \mathrm{L}$ of elution buffer, split into two EP tubes, and stored at $-80^{\circ} \mathrm{C} \mathrm{C}$ until use.

\section{Primer, Probe, and Plasmid Construct Design}

Complete genome sequences of HADV3 and HADV7 were downloaded from the Nation Center for Biotechnology Information database (NCBI,https://www.ncbi.nlm.nih.gov/). The sequences were then aligned using VectorNTI 11.5.1 to locate their conserved regions. The RAA primers for HADV3 and HADV7 as the RAP outer primers were adopted from our previous studies (Wang et al., 2019). The respective RAP innerprimers and TaqMan probes were designed using Oligo7. These primers and probes were synthesized and purified by Shanghai Bioengineering (shanghai, China). Detailed information of the primers and probes sequences is shown in Table1.

The target virus fragments were cloned into vector pUC57 as the plasmids. The plasmids were then quantified using Qubit ${ }^{\mathrm{TM}}$ dsDNA HS Assay Kit (Thermo Fisher Scientific, MA, United States) and transformed as copy numbers. Finally, the plasmids were diluted from $10^{0}-10^{9}$ copies/ $\mu \mathrm{L}$ using TE buffer and stored at $-30^{\circ} \mathrm{C}$ until use. The scheme of RAP is shown in Figure 1.

\section{Recombinase Aided PCR Assay and Sensitivity Analysis}

RAP assay was conducted in one tube and involved two stages. In the first stage, the RAA reaction was performed at $39^{\circ} \mathrm{C}$ in a drop suspended from the inner surface of the tube's lid. In the second stage, qPCR was initiated at the bottom of the tube after spinning down the contents and placing the tube in a thermal cycler. The RAA reaction (total $10 \mu \mathrm{L}$ ) comprised $14 \mathrm{mM}$ of magnesium ion $\left(\mathrm{Mg}^{2+}\right), 200 \mathrm{nM}$ of each RAA primer, $1 \mathrm{uL}$ of template with various concentrations and RAA reaction buffer(Jiangsu, Qitian, Bio-tech Co., Ltd...). The qPCR (total $40 \mu \mathrm{L}$ ) contained $200 \mathrm{nM}$ of each PCR primer and probe, $0.5 \mathrm{mM}$ of each dNTP, and qPCR buffer (Entrans qPCR Probe Set V2, ABclonal, Wuhan, China). RAA was initially performed on the tube's lid, but later, the tube was centrifuged to mix RAA products and qPCR mixture, then incubated at $95^{\circ} \mathrm{C}$ for 5 min to inactivate RAA enzyme and simultaneously begin the denaturation process. The qPCR procedure was as follows: $39^{\circ} \mathrm{C}$ for $10 \mathrm{~min}, 95^{\circ} \mathrm{C}$ for $5 \mathrm{~min}, 95^{\circ} \mathrm{C}$ for $15 \mathrm{~s}, 52.7^{\circ} \mathrm{C}$ for HADV 3 or $48.9^{\circ} \mathrm{C}$ for HADV7 $30 \mathrm{~s}$ and $72^{\circ} \mathrm{C}$ for $30 \mathrm{~s}$.

The sensitivity of the RAP assay was confirmed by performing the assay using serial dilutions of the HADV3 and HADV7 plasmids from $10^{0}$ to $10^{5}$ copies $/ \mu \mathrm{L}$ DNA in nuclease-free water. Meanwhile, we verified the specificity of the RAP assay using clinical samples positive for viruses other than HADV3 and HADV7.

\section{Optimization of Recombinase Aided PCR Assay Working Conditions}

The reaction conditions for RAA and $\mathrm{qPCR}$ were incompatible because the working $\mathrm{Mg}^{2+}$ concentration was significantly different between the two assays. The suitable working concentration of $\mathrm{Mg}^{2+}$ for qPCR was $3 \mathrm{mM}$ while the required concentration of $\mathrm{Mg}^{2+}$ was $14 \mathrm{mM}$ in the RAA reaction mix, which was $4-5$ fold higher than qPCR. As RAA could work well in a volume of $10 \mu \mathrm{L}, 10 \mu \mathrm{L}$ of RAA reaction mix was then added to $30 \mu \mathrm{L}$ or $40 \mu \mathrm{L}$ of qPCR mix. The 
TABLE 1 | Primers and probes sequences used in this study.

\begin{tabular}{|c|c|c|c|}
\hline Assay & Primer/probe & Sequence $\left(5^{\prime}-3^{\prime}\right)$ & Source \\
\hline \multirow[t]{4}{*}{ RAA } & HADV3 outer-F & ATTCCGGCACAGCTTACAATTCACTCGCTCC & Wang et al. (2019) \\
\hline & HADV3 outer-R & TCAGTAGTGG TAATGTCTIT CCCAATTTGC & \\
\hline & HADV7 outer-F & ACAACGGGAGAAGACAATGCCACCACATACAC & \\
\hline & HADV7 outer-R & TCCATCAATATCAGTCCATGATTCTTCTCC & \\
\hline \multirow[t]{6}{*}{ qPCR } & HADV3 inner-F & AGCTTACAATTCACTCGCTCC & This paper \\
\hline & HADV3 inner-R & TAGTGGTAATGTCTITCCCA & \\
\hline & HADV7 inner-F & AGAAGACAATGCCACCACAT & \\
\hline & HADV7 inner-R & CAGTCCATGATTCTTCTCC & \\
\hline & HADV3 P & FAM-ACAATGCAGTAACTACCACCACAAAC-BHQ & \\
\hline & HADV7 P & FAM-AAGACATTACTGCAGACAACAAGCC-BHQ & \\
\hline \multirow[t]{4}{*}{ qPCR } & HADV F primer & GGYCCYAGYTTYAARCCCTAYTC & Qiu et al. (2018) \\
\hline & HADV R primer & AAYTTGAGGYTCTGGYTGATCKG & \\
\hline & HADV3 Probe & HEX-ACAATGCAGTAACTACCACCACAA-MGB & \\
\hline & HADV7 Probe & Cy5-TTACTGCAGACAACAAGCCCAT-MGB & \\
\hline
\end{tabular}
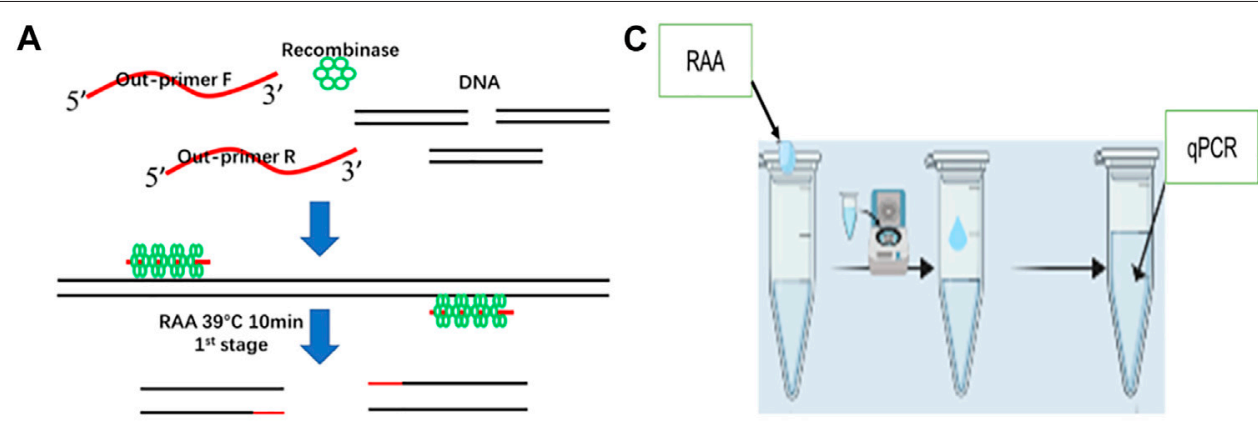

B

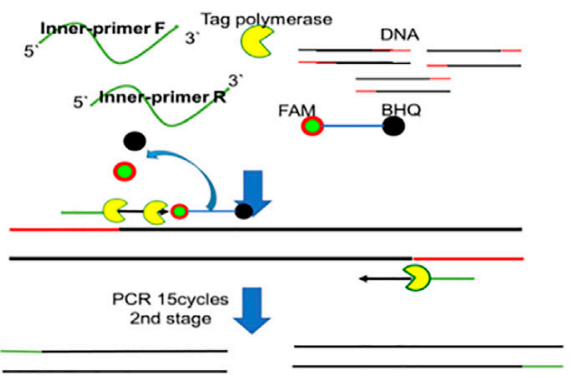

D

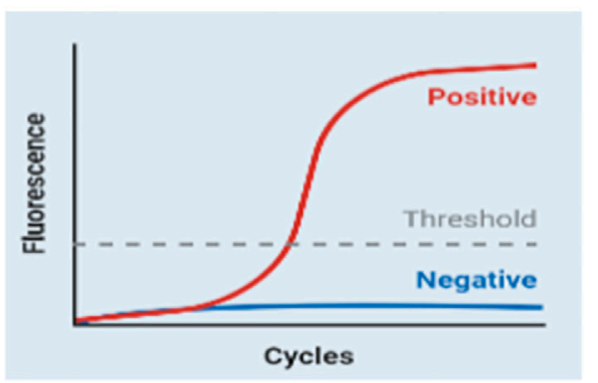

FIGURE 1 | The working principle of Recombinase Aided PCR (RAP) (A): RAA for 10 min at $39^{\circ} \mathrm{C}$ (first stage of RAP). (B): qPCR 15 cycles (second stage of RAP). (C): The reaction tube comprises two compartments. The tube lid (inner surface) compartment contains the RAA mix and the tube bottom compartment houses the qPCR mix. (D): The results can be exhibited by real-time fluorescence curves with qPCR machine.

duration of the RAA reaction was adjusted from 5 to $12 \mathrm{~min}$. The annealing temperature of qPCR was also adjusted from $45^{\circ} \mathrm{C}$ to $65^{\circ} \mathrm{C}$ and the cycle number from 10 to 30 . The outcomes of these adjustments were compared and optimized. The experiments were conducted with $1 \times 10^{4}$ copies $/ \mu \mathrm{L}$ of cDNA. The amplification curve threshold time was served as the figure of merit.

\section{Comparing the Clinical Performance of quantitativePCR Versus Recombinase aided PCR}

Among the 200 stock clinical samples tested using qPCR, 30 were positive for HADV3 and another 30 for HADV7. Subsequently, these samples were simultaneously analyzed using the RAP, and the agreement results were analyzed using SPSS statistics 26 . We further tested $3 \mathrm{HADV} 3$ and $3 \mathrm{HADV} 7$ positive clinical samples that were 4-fold serially diluted using RAP and qPCR.

\section{Quantitative Detection of Recombinase aided PCR}

10-fold serially diluted recombinant plasmids were simultaneously tested using $\mathrm{qPCR}$ and RAP. The experiment was repeated thrice. The CT values of RAP and $\mathrm{qPCR}$ were recorded, and the correlations between RAP and $\mathrm{qPCR}$ were compared using SPSS Statistics 26. 
A

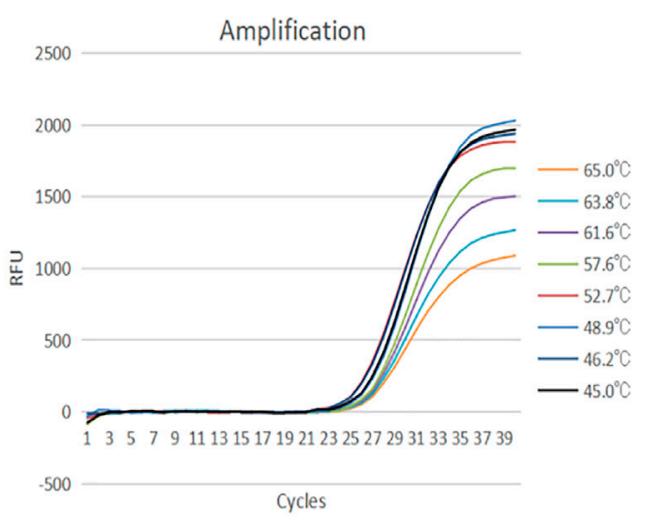

B

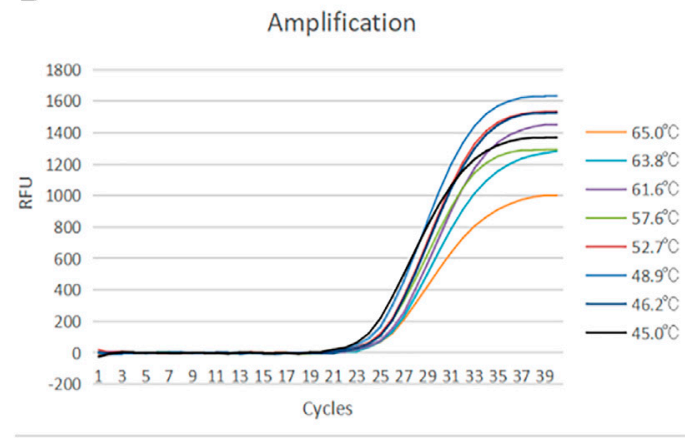

C

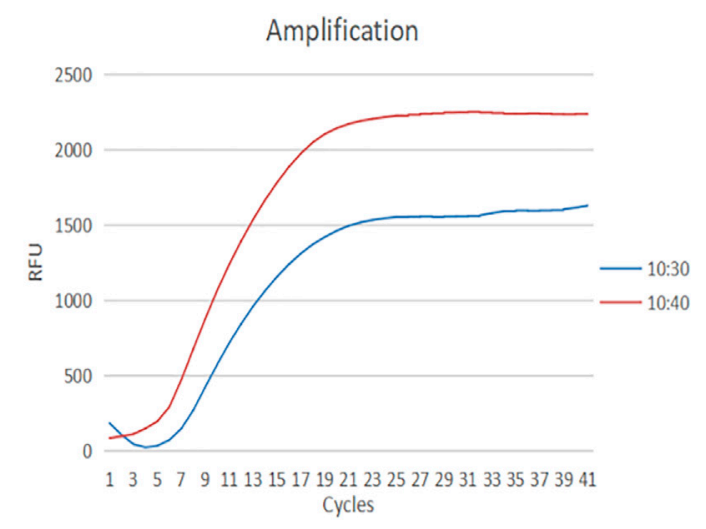

D

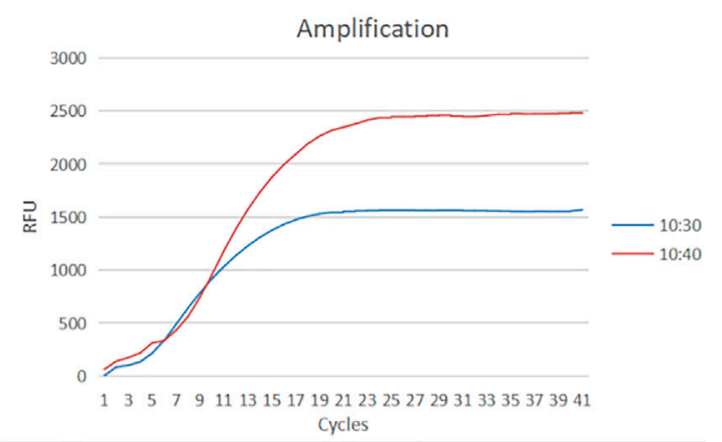

FIGURE 2 | (A) Amplification curve of $\mathrm{HADV} 3$ from $45^{\circ} \mathrm{C}-65^{\circ} \mathrm{C}$, (B) Amplification curve of $\mathrm{HADV} 7$ from $45^{\circ} \mathrm{C}-65^{\circ} \mathrm{C}$. (C) Amplification curve of $\mathrm{HADV} 3$ with a ratio of 1 : 4 and 1:3 of RAA volume to PCR volume. (D) Amplification curve of HADV7 with a ratio of $1: 4$ and 1:3 of RAA volume to PCR volume.

\section{RESULTS}

\section{Recombinase aided PCR Assay Optimization}

The RAP performance was first assessed under different annealing temperatures of $\mathrm{qPCR}$ ranging from 45 to $65^{\circ} \mathrm{C}$. The optimal annealing temperatures for qPCR were $52.7^{\circ} \mathrm{C}$ and $48.9^{\circ} \mathrm{C}$ for $\mathrm{HADV} 3$ and $\mathrm{HADV} 7$, respectively (Figures 2A,B). Secondly, the amplification efficiency of RAP was evaluated by comparing the volume ratio of RAA mix to qPCR mix. According to the $\mathrm{Mg}^{2+}$ concentration difference between RAA and qPCR mix, the ratio of $1: 3$ and $1: 4(10 \mu \mathrm{L}$ RAA mix plus $30 \mathrm{ul}$ or $40 \mu \mathrm{L}$ qPCR mix) were selected. The ratio of $1: 4$ achieved the best amplification efficiency for HADV3 and HADV7 (Figures 2C,D). The RAA assay duration was continuously adjusted from 5 to $12 \mathrm{~min}$ to determine the optimum time for RAA reaction in the first stage of RAP. As the time of RAA reaction increased from 5 to $10 \mathrm{~min}$, the height of the fluorescence curve increased, and the CT value decreased. There was no significant difference between 8 and $10 \mathrm{~min}$, but the height of the fluorescence curve reduced after $10 \mathrm{~min}$ (not shown). The mechanism of reduced height of the fluorescence curve after $10 \mathrm{~min}$ remained unclear. We suspected that some byproducts of RAA reaction might be accumulated along with the time, adversely affecting qPCR.amplification efficiency.
When the different qPCR cycle numbers $(10,15,20,25$, and 30 cycles) were compared, 15 cycles were enough to reach the plateau period in the sensitivity test for HADV3 and HADV7 (Figures 3A,B).

\section{Sensitivity and Specificity of Recombinase Aided PCR Assay}

The sensitivity of the RAP assay for detecting HADV3 and HADV7 was determined using serially diluted recombinant plasmids. A probit analysis established the 95\% limit of HADV3 and HADV7 detection using RAP as 6 and 17 copies per reaction, respectively (Table 2; Figures 3A,B). Meanwhile, the corresponding sensitivity of qPCR was between 100 and 1,000 copies/reaction (Figures 3C,D). No fluorescence signal was detected in any of the negative controls. Out of the 200 stock samples tested, 140 were positive for other respiratory viruses, including human respiratory syncytial virus (HRSV) influenza $A$ and $B$ viruses, rhinovirus, parainfluenza virus, human bocavirus, coronavirus, human metapneumovirus (HMPV), and other HADV species-A (HADV 31), B (HADV 14, 55), C (HADV 1, 2, 5, 6, 57), and E (HADV 4). These samples were also tested to further verify the specificity of RAP. The results of the RAP assay indicated that there is no crossreaction with these pathogens (not shown). 

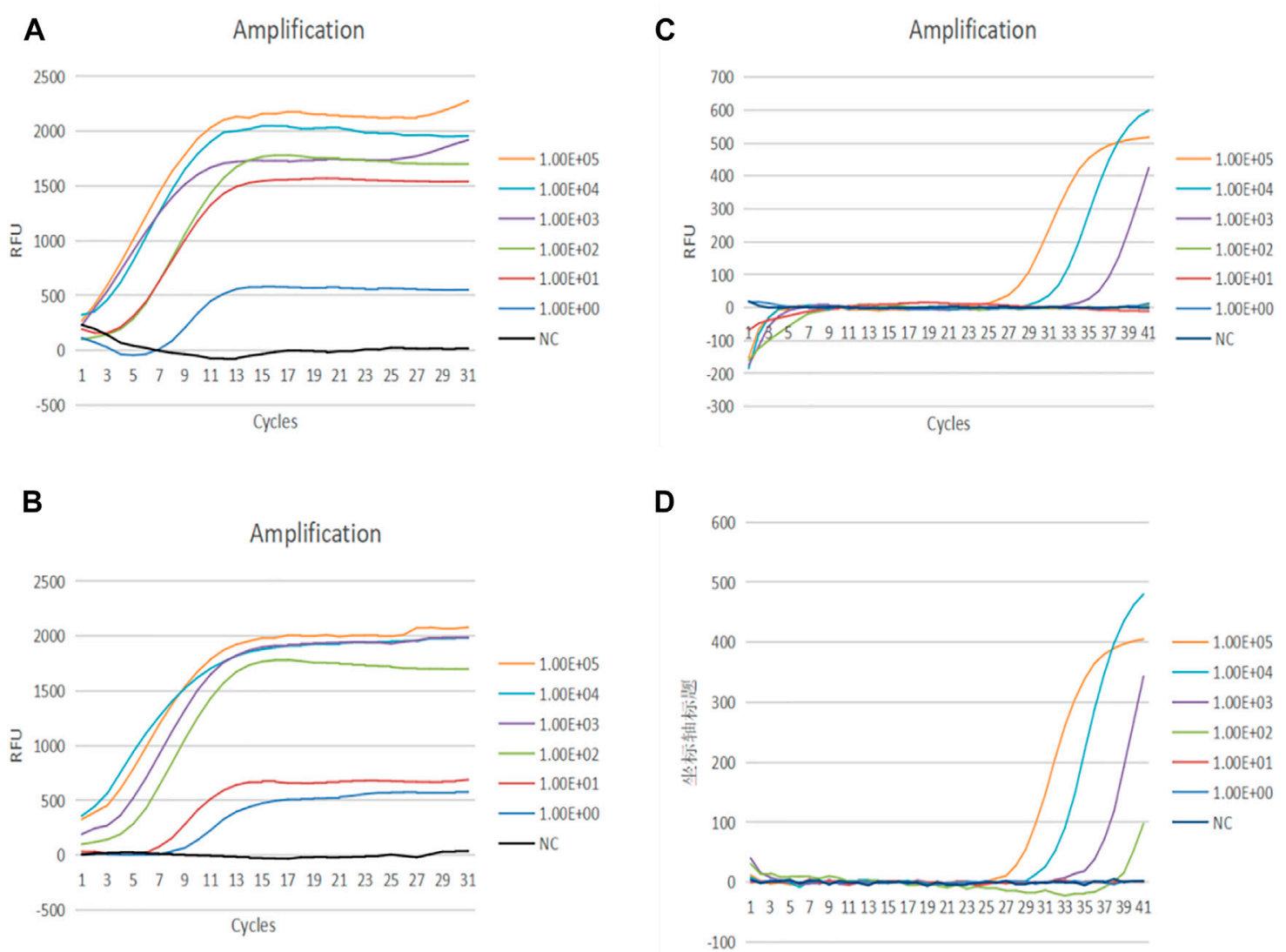

FIGURE 3 | (A) Amplification curve of HADV3 from 100 to 105 copies/reaction using RAP. (B) Amplification curve of HADV7 from $10^{\circ}-10^{5}$ copies/reaction using RAP. (C) Amplification curve of HADV3 from $10^{\circ}-10^{5}$ copies/reaction using qPCR. (D) Amplification curve of HADV7 from $10^{\circ}-10^{5}$ copies/reaction using qPCR.

TABLE 2 | Detection limits of HADV 3 and HADV 7

Copies/reaction

\section{Clinical Performance of Recombinase Aided PCR Versus QuantitativePCR}

The 200 stock samples collected from the patients with respiratory diseases were retested using RAP. These samples were previously tested using qPCR and were found to contain $30 \mathrm{HADV} 3$ positive samples (CT value from 19.2 to 28.9) and 30 ADV7 (CT value from 20.6 to 35.8) positive samples. RAP results were consistent with qPCR, indicating $100 \%$ sensitivity and specificity (Table3). Additionally, we tested three 4-fold serially diluted clinical samples with low CT values (22.0, 22.6, 26.8 for HADV3, and 26.1, 26.0, 20.6 for
No. of positive samples tested by the RAP assay for detection of HADV 3 and HADV 7

HADV3

HADV7

$4 / 8$
$8 / 8$
$8 / 8$
$8 / 8$
$8 / 8$
$8 / 8$

TABLE 3 | Detection of HADV3 and HADV7 in clinical samples.

\begin{tabular}{lcccccc} 
Virus & \multicolumn{2}{c}{ qPCR } & & \multicolumn{2}{c}{ RAP } & Agreement \\
\cline { 2 - 3 } & Positive & Negative & & Positive & Negative & \\
\hline HADV3 & 30 & 170 & & 30 & 170 & $100.00 \%$ \\
HADV7 & 30 & 170 & & 30 & 170 & $100.00 \%$
\end{tabular}

HADV7) using RAP and qPCR. Regarding HADV3 clinical samples, the detection limits of samples 1 and 2 reached $2^{14}$ times dilution at a CT value of 38.1. Meanwhile, sample 3 
reached $2^{10}$ times dilution at a CT value of 36.6 using $\mathrm{qPCR}$. The three clinical samples also tested positive for HADV3 at $2^{18}$ fold dilution using RAP. Hence, RAP was at least 16 -fold $\left(2^{4}\right)$ sensitive than qPCR. Regarding HADV7 clinical samples, RAP was also at least 16 -fold sensitive than qPCR (Supplementary Table S4).

\section{Quantitative Analysis of Recombinase Aided PCR}

Serially diluted $10^{0}-10^{5}$ copies of plasmids were tested to formulate the corresponding quantitative curves of RAP. Based on the results, the curve started to rise at the beginning of qPCR when the initial template concentration was above $10^{3}$ copies. However, no $\mathrm{Ct}$ value was generated at this point, suggesting that a very high concentration of template was accumulated after a $10 \mathrm{~min}$ RAA reaction. In addition, there was no correlation between the plasmids of different concentrations and the corresponding CT values. Therefore, we speculated that the amplification efficiency of RAA was high enough even when the amount of template was low and RAA reaction occurred randomly, resulting in the fluctuation of CT values (Supplementary Table S5).

\section{DISCUSSION AND OUTLOOK}

Respiratory viruses, such as HADV3 and HADV7 cause acute respiratory tract infection (ARTIs), presenting as fever, cough, and pneumonia. These conditions can lead to death in severe cases (Mahony, 2008; Rebelo-de-Andrade et al., 2010; Tregoning and Schwarze, 2010; Mahony et al., 2011; To et al., 2017). Various methods have been applied to detect these viruses, including isothermal nucleic acid amplification and PCR-based amplification technique (Zhao et al., 2015; Lim et al., 2018; Pumford et al., 2020; El-Tholoth et al., 2021; Ganguli et al., 2021; Zhang et al., 2021). RAA can accomplish sample detection in $30 \mathrm{~min}$ with a sensitivity of a single copy; however, the RAA probe is too long (46-52bp) and, therefore, difficult to design. The detection limit of qPCR assay is 100 copies/reaction for both HADV3 and HADV7 ${ }^{[36]}$, which is too low for clinical sample test. $\mathrm{N}$-PCR exhibits the same high sensitivity as RAA but is time consuming (takes more than $2 \mathrm{~h}$ ) (Feng et al., 2018; Zhao et al., 2020).

In this study, we established a novel N-PCR approach, named RAP (recombinase aided PCR) by integrating the merits of RAA and $\mathrm{qPCR}$ detection techniques. RAP is a robust detection method that is more rapid and sensitive than the conventional qPCR. The principle of RAP involves a first-round amplification step using one pair of RAA primers (outer primers) followed by a second round of amplification using one pair of $\mathrm{qPCR}$ primers (inner primers) and a $\mathrm{qPCR}$ probe. The whole process is performed in a single tube. The RAA products are placed in the lid of the tube and briefly centrifuged to mix with the qPCR buffer at the bottom of the tube. The qPCR occurs at the bottom of the tube after heat activation of a heat-start Taq DNA polymerase. Meanwhile, the RAA components get inactivated at this point. RAP capitalizes on the rapid and high amplification efficiency of RAA and the flexibility of TaqMan probe of qPCR to detect pathogens. It avoids the design of a complex RAA probe. RAP assay for respiratory DNA viruses (HADV3 and HADV7) demonstrated detection limits of 6 and 17.0copies per reaction for $\mathrm{HADV} 3$ and $\mathrm{HADV} 7$, respectively (probit analysis $p \leq 0.05)$, which are lower than those of corresponding qPCR. The results of clinical application of RAP in testing 200 samples were consistent with those of qPCR. Of note, no cross-reaction was observed with other common respiratory virus-positive samples. The testing results using serially diluted clinical samples also indicated that RAP possesses higher sensitivity. The rapidity of RAP ( $1 \mathrm{~h}$ and single-tube assay) protects the reaction from contaminating and saves running time compared to qPCR. RAP does not depend on the isothermal detection device since it can be performed on a conventional real-time thermal cycler, indicating that it can be applied in general laboratories. Overlapping of $\mathrm{qPCR}$ primers with RAA primers was explored in our preliminary study to further simplify the design of RAP primers. And no adverse impact on the RAP amplification efficiency was observed.

However, RAP has some limitations. Although the RAP analysis result is shown by CT value of $\mathrm{qPCR}$, the CT value cannot quantify the virus titer in the original samples. Because of the extremely high amplification efficiency of RAA, most respiratory clinical samples generate no or very low CT values in the second stage of the qPCR, suggesting that the amplification for most samples reaches the plateau phase after RAA. Further improvement of RAP will aim to 1) use a Taq enzyme that can function in the RAA reaction mix containing a high $\mathrm{Mg}^{2+}$ concentration to allow the use of only one mix in the tube. 2) develop multiple detections of pathogens. 3) reduce RAA reaction time and increase qPCR cycles for quantitative detection of pathogens.

Despite the few limitations of RAP in this proof-of-concept study, RAP can serve as a robust, rapid, and highly sensitive detection platform with a wide potential application in the detection of DNA/RNA viruses, bacteria, and SNPs. In particular, RAP can be used for fast screening of newly infected asymptomatic patients, sensitive detection of cerebrospinal fluid samples with low viral load, monitoring low frequency of drug resistance pathogens, such as Mycobacterium tuberculosis, and monitoring the treatment efficacy of HIV patients with low viral load.

Our study demonstrated that the RAP method enables a rapid and highly sensitive detection using a conventional qPCR device. The merits of RAP are the high sensitivity and adaptability, making RAP a great potential to be cooperated with existing qPCR and microfluidic devices. Further attempts will be made to integrate RAP with microfluidic technology and develop a biosensor-based instrument towards clinical and point-of-care applications in the rapid, field detection of multiplex pathogens in the future. 


\section{DATA AVAILABILITY STATEMENT}

The original contributions presented in the study are included in the article/Supplementary Material, further inquiries can be directed to the corresponding authors.

\section{AUTHOR CONTRIBUTIONS}

XM, XS designed the study. GF, RZ, FT, MN performed the experiments. GF, RZ, XH analyzed and interpreted the data. XM, GF wrote the paper. All authors provided a critical review and approved the final article.

\section{FUNDING}

This work was supported by grants from IVDC (2019HYDQNJJ03), China Mega-Projects for Infectious

\section{REFERENCES}

Bonroy, C., Vankeerberghen, A., Boel, A., and De Beenhouwer, H. (2007). Use of a Multiplex Real-Time PCR to Study the Incidence of Human Metapneumovirus and Human Respiratory Syncytial Virus Infections During Two winter Seasons in a Belgian Paediatric Hospital. Clin. Microbiol. Infect. 13 (5), 504-509. doi:10.1111/j.1469-0691.2007.01682.x

Chen, Y., Mei, Y., Zhao, X., and Jiang, X. (2020). Reagents-Loaded, Automated Assay that Integrates Recombinase-Aided Amplification and Cas12a Nucleic Acid Detection for a Point-of-Care Test. Anal. Chem. 92 (21), 14846-14852. doi:10.1021/acs.analchem.0c03883

Corman, V. M., Landt, O., Kaiser, M., Molenkamp, R., Meijer, A., Chu, D. K., et al. (2020). Detection of 2019 Novel Coronavirus (2019-nCoV) by Real-Time RTPCR. Euro Surveill. 25 (3), 2000045. doi:10.2807/15607917.ES.2020.25.3.2000045

Duan, S., Li, G., Li, X., Chen, C., Yan, T., Qiu, F., et al. (2018). A Probe Directed Recombinase Amplification Assay for Detection of MTHFR A1298C Polymorphism Associated With Congenital Heart Disease. Biotechniques. 64 (5), 211-217. doi:10.2144/btn-2018-2010

El-Tholoth, M., Anis, E., and Bau, H. H. (2021). Two Stage, Nested Isothermal Amplification in a Single Tube. Analyst. 146 (4), 1311-1319. doi:10.1039/ d0an01835j

Fan, G. H., Shen, X. X., Li, F., Li, X. N., Bai, X. D., Zhang, R. Q., et al. (2019). Development of an Internally Controlled Reverse Transcription RecombinaseAided Amplification Assay for the Rapid and Visual Detection of West Nile Virus. Biomed. Environ. Sci. 32 (12), 926-929. doi:10.3967/bes2019.116

Feng, Z.-s., Zhao, L., Wang, J., Qiu, F.-z., Zhao, M.-c., Wang, L., et al. (2018). A Multiplex One-Tube Nested Real Time RT-PCR Assay for Simultaneous Detection of Respiratory Syncytial Virus, Human Rhinovirus and Human Metapneumovirus. Virol. J. 15 (1), 167. doi:10.1186/s12985-018-1061-0

Ganguli, A., Mostafa, A., Berger, J., Lim, J., Araud, E., Baek, J., et al. (2021). Reverse Transcription Loop-Mediated Isothermal Amplification Assay for Ultrasensitive Detection of SARS-CoV-2 in Saliva and Viral Transport Medium Clinical Samples. Anal. Chem. 93 (22), 7797-7807. doi:10.1021/ acs.analchem.0c05170

Kellner, M. J., Koob, J. G., Gootenberg, J. S., Abudayyeh, O. O., and Zhang, F. (2019). SHERLOCK: Nucleic Acid Detection With CRISPR Nucleases. Nat. Protoc. 14 (10), 2986-3012. doi:10.1038/s41596-019-0210-2

Lim, D.-R., Kim, H.-R., Park, M.-J., Chae, H.-G., Ku, B.-K., Nah, J.-J., et al. (2018). A Tailored Reverse Transcription Loop-Mediated Isothermal Amplification for Sensitive and Specific Detection of Serotype A Foot-And-Mouth Disease Virus Circulating in Pool 1 Region Countries. Transbound Emerg. Dis. 65 (6), 1898-1908. doi:10.1111/tbed.12971
Disease (2018ZX10711001,2017ZX10104001 and 2018ZX10713-002), A Chinese patent (202110073720.0) is pending.

\section{ACKNOWLEDGMENTS}

We gratefully acknowledge the assistance from National Institute for Viral Disease Control and Prevention (IVDC).Chinese Center for Disease Control and Prevention (CCDC).

\section{SUPPLEMENTARY MATERIAL}

The Supplementary Material for this article can be found online at: https://www.frontiersin.org/articles/10.3389/fbioe.2021.766411/ full\#supplementary-material

Liu, M., Li, C.-c., Luo, X., Ma, F., and Zhang, C.-y. (2020). 5 Hydroxymethylcytosine Glucosylation-Triggered Helicase-dependent Amplification-Based Fluorescent Biosensor for Sensitive Detection of $\beta$-Glucosyltransferase With Zero Background Signal. Anal. Chem. 92 (24), 16307-16313. doi:10.1021/acs.analchem.0c04382

Lobato, I. M., and O'Sullivan, C. K. (2018). Recombinase Polymerase Amplification: Basics, Applications and Recent Advances. Trac Trends Anal. Chem. 98, 19-35. doi:10.1016/j.trac.2017.10.015

Mahony, J. B., Petrich, A., and Smieja, M. (2011). Molecular Diagnosis of Respiratory Virus Infections. Crit. Rev. Clin. Lab. Sci. 48 (5-6), 217-249. doi:10.3109/10408363.2011.640976

Mahony, J. B. (2008). Detection of Respiratory Viruses by Molecular Methods. Clin. Microbiol. Rev. 21 (4), 716-747. doi:10.1128/cmr.00037-07

Marano, J. M., Chuong, C., and Weger-Lucarelli, J. (2020). Rolling circle Amplification: A High Fidelity and Efficient Alternative to Plasmid Preparation for the rescue of Infectious Clones. Virology. 551, 58-63. doi:10.1016/j.virol.2020.08.016

Paul, R., Ostermann, E., and Wei, Q. (2020). Advances in Point-of-Care Nucleic Acid Extraction Technologies for Rapid Diagnosis of Human and Plant Diseases. Biosens. Bioelectron. 169, 112592. doi:10.1016/ j.bios.2020.112592

Pumford, E. A., Lu, J., Spaczai, I., Prasetyo, M. E., Zheng, E. M., Zhang, H., et al. (2020). Developments in Integrating Nucleic Acid Isothermal Amplification and Detection Systems for Point-of-Care Diagnostics. Biosens. Bioelectron. 170, 112674. doi:10.1016/j.bios.2020.112674

Qi, J., Li, X., Zhang, Y., Shen, X., Song, G., Pan, J., et al. (2019). Development of a Duplex Reverse Transcription Recombinase-Aided Amplification Assay for Respiratory Syncytial Virus Incorporating an Internal Control. Arch. Virol. 164 (7), 1843-1850. doi:10.1007/s00705-019-04230-Z

Qiu, F.-z., Shen, X.-x., Zhao, M.-c., Zhao, L., Duan, S.-x., Chen, C., et al. (2018). A Triplex Quantitative Real-Time PCR Assay for Differential Detection of Human Adenovirus Serotypes 2, 3 and 7. Virol. J. 15 (1), 81. doi:10.1186/ s12985-018-0983-x

Rebelo-de-Andrade, H., Pereira, C., Gi'ria, M., Prudêncio, E., Brito, M. J., Cale', E., et al. (2010). Outbreak of Acute Respiratory Infection Among Infants in Lisbon, Portugal, Caused by Human Adenovirus Serotype 3 and a New $7 / 3$ Recombinant Strain. J. Clin. Microbiol. 48 (4), 1391-1396. doi:10.1128/ jcm.02019-09

Riley, L. W. (2018). Laboratory Methods in Molecular Epidemiology: Bacterial Infections. Microbiol. Spectr. 6 (6). doi:10.1128/microbiolspec.AME-0004-2018

Song, J., Liu, C., Mauk, M. G., Rankin, S. C., Lok, J. B., Greenberg, R. M., et al. (2017). Two-Stage Isothermal Enzymatic Amplification for Concurrent Multiplex Molecular Detection. Clin. Chem. 63 (3), 714-722. doi:10.1373/ clinchem.2016.263665 
To, K. K., Lu, L., Yip, C. C., Poon, R. W., Fung, A. M., Cheng, A., et al. (2017). Additional Molecular Testing of Saliva Specimens Improves the Detection of Respiratory Viruses. Emerg. Microbes Infect. 6 (6), e49. doi:10.1038/emi.2017.35

Tregoning, J. S., and Schwarze, J. (2010). Respiratory Viral Infections in Infants: Causes, Clinical Symptoms, Virology, and Immunology. Clin. Microbiol. Rev. 23 (1), 74-98. doi:10.1128/cmr.00032-09

Tu, F., Yang, X., Xu, S., Chen, D., Zhou, L., Ge, X., et al. (2020). Development of a Fluorescent Probe-Based Real-Time Reverse Transcription RecombinaseAided Amplification Assay for the Rapid Detection of Classical Swine Fever Virus. Transbound Emerg. Dis. 68 (4), 2017-2027. doi:10.1111/tbed.13849

Waggoner, J. J., Gresh, L., Mohamed-Hadley, A., Ballesteros, G., Davila, M. J. V., Tellez, Y., et al. (2016). Single-Reaction Multiplex Reverse Transcription PCR for Detection of Zika, Chikungunya, and Dengue Viruses. Emerg. Infect. Dis. 22 (7), 1295-1297. doi:10.3201/eid2207.160326

Wang, J., Cai, K., He, X., Shen, X., Wang, J., Liu, J., et al. (2020). Multiple-Centre Clinical Evaluation of an Ultrafast Single-Tube Assay for SARS-CoV-2 RNA. Clin. Microbiol. Infect. 26 (8), 1076-1081. doi:10.1016/j.cmi.2020.05.007

Wang, R.-h., Zhang, H., Zhang, Y., Li, X.-n., Shen, X.-X., Qi, J.-j., et al. (2019). Development and Evaluation of Recombinase-Aided Amplification Assays Incorporating Competitive Internal Controls for Detection of Human Adenovirus Serotypes 3 and 7. Virol. J. 16 (1), 86. doi:10.1186/s12985-019-1178-9

Zhang, C., Zheng, T., Wang, H., Chen, W., Huang, X., Liang, J., et al. (2021). Rapid One-Pot Detection of SARS-CoV-2 Based on a Lateral Flow Assay in Clinical Samples. Anal. Chem. 93 (7), 3325-3330. doi:10.1021/acs.analchem.0c05059

Zhang, R.-q., Li, G.-x., Li, X.-n., Shen, X.-x., Gao, Y., Wang, L., et al. (2019). A Rapid and Sensitive Recombinase Aided Amplification Assay Incorporating Competitive Internal Control to Detect Bordetella Pertussis Using the DNA Obtained by Boiling. Int. J. Infect. Dis. 86, 108-113. doi:10.1016/ j.ijid.2019.06.028

Zhang, R.-q., Li, Z., Li, G.-x., Tie, Y.-q., Li, X.-n., Gao, Y., et al. (2020). A Highly Sensitive One-Tube Nested Quantitative Real-Time PCR Assay for Specific
Detection of Bordetella Pertussis Using the LNA Technique. Int. J. Infect. Dis. 93, 224-230. doi:10.1016/j.ijid.2020.01.053

Zhao, L., Li, G-X., Wang, J., Zhao, M-C., Wang, L., Feng, Z-S., et al. (2020). Development and Evaluation of a Panel of Multiplex One-Tube Nested Real Time PCR Assay for Simultaneous Detection of 14 Respiratory Viruses in Five Reactions. J. Med. Virol. doi:10.1002/jmv.25686

Zhao, Y., Chen, F., Li, Q., Wang, L., and Fan, C. (2015). Isothermal Amplification of Nucleic Acids. Chem. Rev. 115 (22), 12491-12545. doi:10.1021/ acs.chemrev.5b00428

Zhu, X., Wang, X., Han, L., Chen, T., Wang, L., Li, H., et al. (2020). Multiplex Reverse Transcription Loop-Mediated Isothermal Amplification Combined with Nanoparticle-Based Lateral Flow Biosensor for the Diagnosis of COVID-19. Biosens. Bioelectron. 166, 112437. doi:10.1016/j.bios.2020.112437

Conflict of Interest: The authors declare that the research was conducted in the absence of any commercial or financial relationships that could be construed as a potential conflict of interest.

Publisher's Note: All claims expressed in this article are solely those of the authors and do not necessarily represent those of their affiliated organizations, or those of the publisher, the editors, and the reviewers. Any product that may be evaluated in this article, or claim that may be made by its manufacturer, is not guaranteed or endorsed by the publisher.

Copyright (c) 2021 Fan, Zhang, He, Tian, Nie, Shen and Ma. This is an open-access article distributed under the terms of the Creative Commons Attribution License (CC $B Y)$. The use, distribution or reproduction in other forums is permitted, provided the original author(s) and the copyright owner(s) are credited and that the original publication in this journal is cited, in accordance with accepted academic practice. No use, distribution or reproduction is permitted which does not comply with these terms. 